\title{
High-quality stable electron beams from laser wakefield acceleration in high density plasma
}

\author{
B. S. Rao, ${ }^{*}$ A. Moorti, R. Rathore, J. A. Chakera, P. A. Naik, and P. D. Gupta \\ Laser Plasma Division, Raja Ramanna Centre for Advanced Technology, Indore 452013, India
}

(Received 12 July 2013; published 8 January 2014)

\begin{abstract}
High-quality, stable electron beams are produced from self-injected laser wakefield acceleration using the interaction of moderate $3 \mathrm{TW}, 45$ fs duration Ti:sapphire laser pulses with high density $\left(>5 \times 10^{19} \mathrm{~cm}^{-3}\right)$ helium gas jet plasma. The electron beam has virtually background-free quasimonoenergetic distribution with energy $35.6_{-2.5}^{+3.9} \mathrm{MeV}$, charge $3.8_{-1.2}^{+2.8} \mathrm{pC}$, divergence and pointing variation $\sim 10 \mathrm{mrad}$. The stable and high quality of the electron beam opens an easy way for applications of the laser wakefield accelerator in the future, particularly due to the widespread availability of sub-10 TW class lasers with a number of laser plasma laboratories around the world.
\end{abstract}

DOI: 10.1103/PhysRevSTAB.17.011301

PACS numbers: 41.75.Jv, 52.59.Bi, 52.59.Fn

Laser wakefield acceleration is currently widely pursued as a promising technique to realize compact high-energy electron accelerators in the future [1]. Extensive investigations of this technique, particularly over the past decade, have resulted in the generation of high-quality electron bunches with energies up to $1 \mathrm{GeV}$ from tabletop setups operating in the "blowout" regime. In the blowout" regime [2], which is also known as the "bubble" regime, a relativistically intense, ultrashort laser pulse propagating in underdense plasma blows out the plasma electrons radially by the ponderomotive force forming a near spherical electron bubble that propagates with the laser pulse with a velocity close to $c$. The bubble formation is most efficient when the pulse dimensions, i.e., focal spot $\left(w_{0}\right)$ and pulse length $\left(c \tau_{L}\right)$, are on the order of plasma wavelength $\left(\lambda_{p}\right)$. When the driver laser pulse is intense enough, it can lead to self-injection of ambient plasma electrons. The electrons get accelerated by the strong axial electric field of the bubble, resulting in the generation of an energetic electron beam. In order to be useful for potential applications [3], the accelerated electron beam should be stable (shot to shot) and should have low divergence, energy spread, and pointing variation. In addition, electron beams without any low-energy background electrons are desirable for high signal-to-noise ratio in the electron beam and $\mathrm{x}$-ray interaction studies and to reduce the unwanted, potentially hazardous bremsstrahlung radiation. Stable and controllable electron beams have been experimentally demonstrated using various schemes of localized injection [4-7]. Some of these experiments have reported quasimonoenergetic electron beams with less or

\footnotetext{
*sunnyb@rrcat.gov.in

Published by the American Physical Society under the terms of the Creative Commons Attribution 3.0 License. Further distribution of this work must maintain attribution to the author(s) and the published articles title, journal citation, and DOI.
}

virtually no low-energy background electrons. In order to generate such beams, the time duration of self-injection must be much shorter than the laser pulse transit time through the plasma. Theory and simulations suggest that controlling the evolution of the driving laser pulse can improve localization of the electron injection [8]. Alternatively, operating the laser wakefield accelerator near the self-injection threshold could also limit the duration of self-injection. Recent studies in the bubble regime, using 40-50 TW power or few cycle laser pulses, and plasma density $\leq 2 \times 10^{19} \mathrm{~cm}^{-3}\left(c \tau_{L} \leq \lambda_{p}\right)$, have shown self-injected quasimonoenergetic electron beams of $\sim 300 \mathrm{MeV}$ or $25 \mathrm{MeV}$, with apparently no low-energy background electrons $[9,10]$.

In this paper, we report the demonstration of stable and high-quality electron beams from self-injected laser wakefield acceleration, using a relatively much lower power (3 TW) and longer duration (45 fs) Ti:sapphire laser pulses interacting with high density $\left(5.8 \times 10^{19} \mathrm{~cm}^{-3}\right)$ plasma. Background-free, quasimonoenergetic beams with central energy around $35 \mathrm{MeV}$ and few picocoulomb (pC) charge were produced. The observations suggest that rapid selfmodulation and self-evolution of the laser pulse assisted by the interaction at high plasma density could eventually provide access to the blowout regime although initial laser parameters are far from the matched values. The high quality of the electron beam production indicates operation of the laser wakefield accelerator near self-injection threshold.

A schematic diagram of the experimental setup is shown in Fig. 1. The experiment was performed using a Ti:sapphire laser system that delivers horizontally polarized laser pulses of full width at half maximum (FWHM) duration $\tau_{L} \sim 45 \mathrm{fs}$, having central wavelength $\lambda_{0} \simeq 800 \mathrm{~nm}$. The laser beam was focused using an $\mathrm{f} / 5$ off-axis parabola, along $1.2 \mathrm{~mm}$ width of a rectangular supersonic helium gas jet, such that the focus was at a height of $1 \mathrm{~mm}$ above the nozzle orifice. The gas jet density was obtained from 


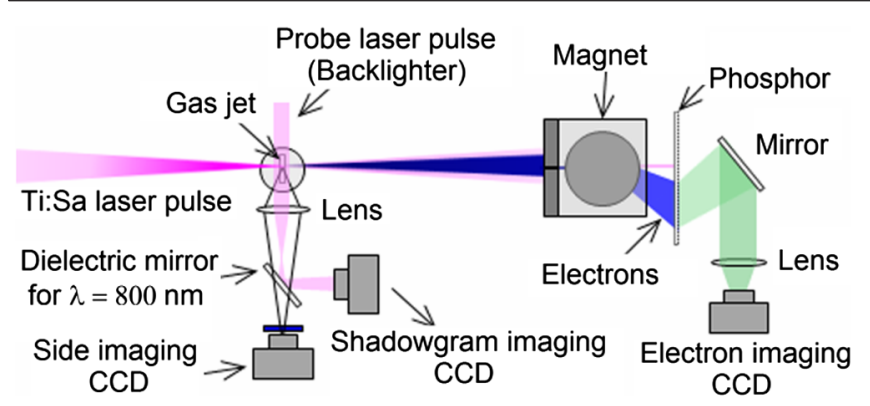

FIG. 1 (color online). A schematic diagram of the experimental setup.

interferometry and it was further confirmed from the forward Raman scattering measurements [11,12]. The longitudinal profile of the gas jet was flat topped, with scale length about $100 \mu \mathrm{m}$ at the edges. Backing pressure of the gas jet was varied to adjust the electron density $\left(n_{e}\right)$ of the helium plasma to be in the range of $10^{19}-10^{20} \mathrm{~cm}^{-3}$. The full width at $1 / \mathrm{e}^{2}$ maximum size of the focal spot was $27 \mu \mathrm{m} \times 15 \mu \mathrm{m}$, and was approximated with an equivalent area circular spot of $1 / \mathrm{e}^{2}$ width, $2 w_{0}=20 \mu \mathrm{m}$ to estimate the peak intensity. The peak power of the laser pulse on the target was $P_{L}=0.92 E_{L} / \tau_{L} \simeq 3$ TW considering $E_{L}=145 \mathrm{~mJ}$ contained in the focal spot and assuming a Gaussian temporal profile. The peak intensity in the focal spot was estimated to be $I_{L}=2 P_{L} / \pi w_{0}^{2}=$ $2 \times 10^{18} \mathrm{~W} / \mathrm{cm}^{2}\left(a_{0} \simeq 1\right)$. For measuring the energy spectrum, a single-shot electron spectrograph consisting of two circular permanent magnets with $50 \mathrm{~mm}$ diameter, $9 \mathrm{~mm}$ pole gap, and effective magnetic field $B_{\text {eff }}=0.46 \mathrm{~T}$, was used to deflect the electrons onto a $\mathrm{Gd}_{2} \mathrm{O}_{2} \mathrm{~S}: \mathrm{Tb}$ phosphor screen (model DRZ-high) that was imaged with a 16-bit charge coupled device (CCD) camera. A slit was used at the entrance of the magnet to allow in only the electrons within $8 \mathrm{mrad}$ acceptance angle in the plane of electron dispersion. The low-energy cutoff of the electron spectrometer was $10 \mathrm{MeV}$. The electron beam charge was estimated using the absolute calibration data of the phosphor screen [13]. The electron beam profile and the pointing variation were measured by moving the magnet out from the path of the electron beam. The laser-plasma interaction was imaged from the nonlinear Thomson side-scattering radiation at second harmonic $(400 \mathrm{~nm})$ of the laser, with $5 \times$ magnification using a 12-bit CCD camera. A narrow bandpass filter with transmission wavelength at $400 \pm 20 \mathrm{~nm}$ was kept in front of the CCD camera for collecting the second harmonic radiation. A small fraction of the drive laser beam, with variable delay relative to the main laser beam, was used as a probe beam (backlighter) to record the shadowgram images of the interaction region with 2 ps time resolution. The probe beam was sent perpendicular to the incident laser axis and the gas jet flow direction and was in the direction of the side-imaging setup. A multilayered dielectric mirror, with high reflectivity at
$800 \mathrm{~nm}$ radiation, was placed in front of the side imaging CCD camera to reflect the probe beam onto a separate 12bit CCD camera, while allowing the $400 \mathrm{~nm}$ radiation to reach the side imaging CCD camera, as shown in Fig. 1. A narrow bandpass filter that allows the radiation with wavelength within $800 \pm 20 \mathrm{~nm}$ was kept in front of the shadowgram imaging CCD camera.

After scanning the plasma density by adjusting the backing pressure of the gas jet, and fine-tuning the laser focus position in the gas jet, the electron signal was observed on the phosphor for plasma density $n_{e} \simeq 3.6 \times 10^{19} \mathrm{~cm}^{-3}$. However, the typical electron beam had low charge ( $\ll 1 \mathrm{pC}$ ), large divergence ( $>20 \mathrm{mrad})$, and poor reproducibility. As the density was increased, at $n_{e} \simeq 5.8 \times 10^{19} \mathrm{~cm}^{-3}$, a highly stable, low divergence electron beam without any background halo was observed, in contrast to most of the earlier experiments, particularly at high plasma density $\left(>2 \times 10^{19} \mathrm{~cm}^{-3}\right)$ [1]. When the plasma density was increased beyond $n_{e} \simeq 6 \times 10^{19} \mathrm{~cm}^{-3}$, a collimated electron beam with high spatial contrast was still present but the divergence increased with density, as shown in Fig. 2. The electron beam produced at $n_{e} \simeq 5.8 \times 10^{19} \mathrm{~cm}^{-3}$ was highly reproducible with $>90 \%$ probability, in a series of about 10-20 shots during most of the experimental runs. As seen in Fig. 2(a), the transverse profile of the electron beam at $n_{e} \simeq 5.8 \times 10^{19} \mathrm{~cm}^{-3}$ was elliptical in shape with its major axis along the horizontal direction, which was the direction of laser polarization. The ellipticity (defined here as the ratio of the length of major axis to minor axis) of the electron beam was estimated to be $\varepsilon=1.9_{-0.3}^{+0.3}$. The FWHM divergence of the electron beam was $\Delta \theta_{X}=13.1_{-2.0}^{+4.6} \mathrm{mrad}$ in the horizontal direction and $\Delta \theta_{Y}=7.2_{-0.7}^{+2.8} \mathrm{mrad}$ in the vertical direction. The errors shown are the root-mean-square (rms) deviations from the average value. To account for the asymmetric distributions, the rms value was independently calculated for the occurrences with higher-than-average and lower-than-average values. The reproducibility and pointing variation of the electron beam were observed to degrade with increasing plasma densities. The rms pointing

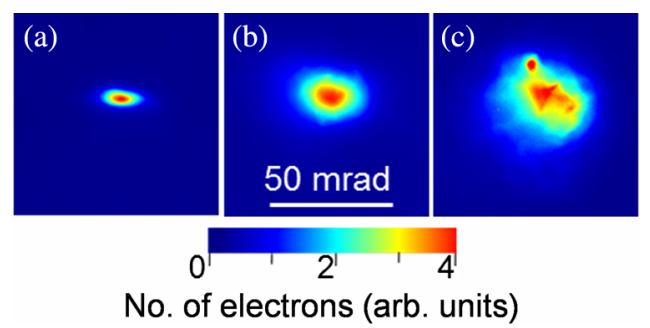

FIG. 2 (color online). Images of the electron beam on the DRZ phosphor screen for varying plasma density: (a) $5.8 \times 10^{19} \mathrm{~cm}^{-3}$, (b) $6.8 \times 10^{19} \mathrm{~cm}^{-3}$, and (c) $8.6 \times 10^{19} \mathrm{~cm}^{-3}$. The images show well-defined profiles with no background halo, particularly at lower density. The FWHM divergences of the electron beam in the horizontal direction are $13 \mathrm{mrad}, 26 \mathrm{mrad}$, and $33 \mathrm{mrad}$ for the images (a), (b), and (c), respectively. 


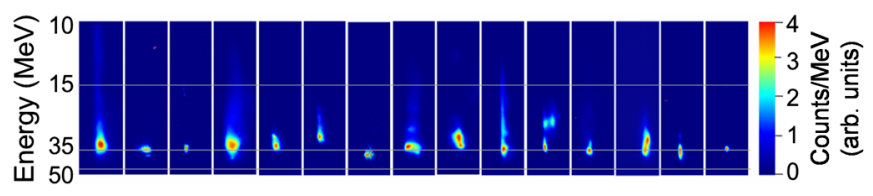

FIG. 3 (color online). Images of energy dispersed electron beams (on the phosphor) produced in 15 consecutive laser shots, at an electron density of $\sim 5.8 \times 10^{19} \mathrm{~cm}^{-3}$ in helium plasma. The color scale is normalized for each shot.

deviation from the mean pointing angle of the electron beam in horizontal and vertical direction was $\left\langle\theta_{X}\right\rangle=12.3 \mathrm{mrad}$ and $\left\langle\theta_{Y}\right\rangle=7.6 \mathrm{mrad}$, respectively. It may be noted that the pointing variation of the electron beam in the horizontal and vertical directions is almost equal to the divergence in respective directions.

A series of images of the electron beam energy spectra recorded at $n_{e} \simeq 5.8 \times 10^{19} \mathrm{~cm}^{-3}$ during one of the experimental runs is shown in Fig. 3. Good shot-to-shot stability was obtained in the central energy (energy at the peak charge density) of the electron bunch. The mean peak energy of the electron beam was $\mathrm{E}_{\text {peak }}=35.6_{-2.5}^{+3.9} \mathrm{MeV}$. The average charge of the electron beam was estimated to be $3.8_{-1.2}^{+2.8} \mathrm{pC}$. Majority of the spectra showed virtually background-free, quasimonoenergetic peak. A typical lineout of the electron spectrum is shown in Fig. 4. The horizontal bar at the quasimonoenergetic peak represents the uncertainty in the peak energy. This uncertainty arises due to the pointing variation of the electron beam in the dispersion plane. Since the acceptance angle $(8 \mathrm{mrad})$ of the slit in front of the spectrograph was smaller than the pointing variation (12.3 $\mathrm{mrad}$ ) of the beam in the dispersion plane, the actual value of the uncertainty is decided by the acceptance angle of the spectrograph. A histogram of the peak energy distribution of the electron beam is shown in Fig. 5. The relative FWHM energy spread $\left(\Delta \mathrm{E}_{\mathrm{FWHM}} / \mathrm{E}_{\text {peak }}\right)$

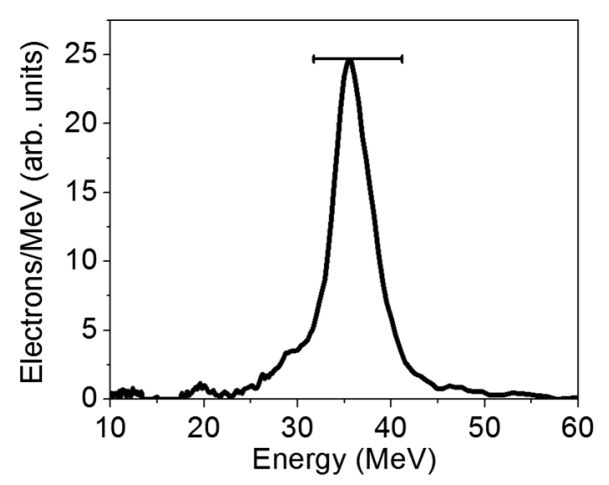

FIG. 4. A lineout energy spectrum of a typical electron beam at an electron density of $\sim 5.8 \times 10^{19} \mathrm{~cm}^{-3}$ in helium plasma, showing almost no low-energy background. The horizontal line on top of the peak shows the uncertainty in the peak energy due to pointing variation within the $8 \mathrm{mrad}$ acceptance angle of the electron spectrograph.

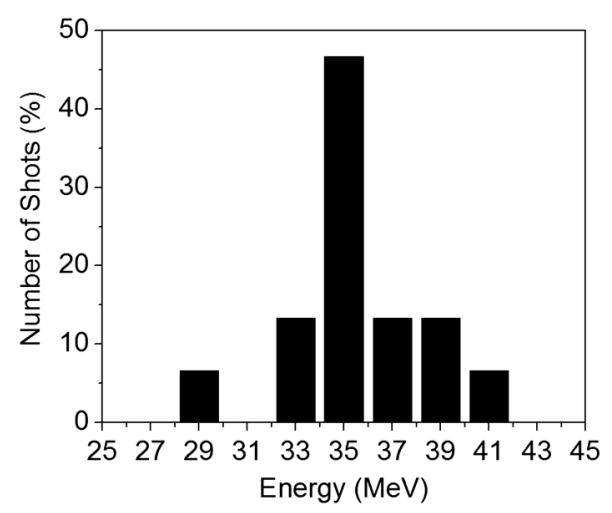

FIG. 5. A histogram of the peak energy of the quasimonoenergetic electron beams produced at an electron density of $\sim 5.8 \times$ $10^{19} \mathrm{~cm}^{-3}$ in helium plasma. The histogram shows 7 bins, each of $2 \mathrm{MeV}$ width.

of the electron beam was estimated to be $29.0_{-11.2}^{+9.6} \%$. Since the average horizontal divergence angle of the electron beam $(13.1 \mathrm{mrad})$ is larger than the acceptance angle $(8 \mathrm{mrad})$ of the spectrometer, the maximum angular dimension of the beam on the phosphor screen in case of single energy electrons would be $\sim 8 \mathrm{mrad}$. This finite size of the beam in the dispersion plane causes an apparent energy spread of $26.7 \%$ about the mean peak energy $\left(\left\langle\mathrm{E}_{\text {peak }}\right\rangle=35.6 \mathrm{MeV}\right)$, which is very close to the measured mean energy spread. Energy spread higher than the mean value occurred for electron beams with long low-energy tails in some shots. In a few shots in Fig. 3, only a small portion at the edge of the electron beam was intercepted by the slit, resulting in a reduced horizontal beam size on the phosphor. Relative energy spread of about $14 \%$ was measured with such images. The shadowgram images recorded at about 2 ps before the arrival of the driver laser pulse showed no preplasma in the gas jet. This indicates that the intensity of the prepulse pedestal due to amplified spontaneous emission in front of the femtosecond laser pulse was below the ionization threshold for helium. Simultaneous measurement of nonlinear Thomson side-scattering images showed smooth and stable selfguiding channels with length $490 \mu \mathrm{m}$. Increasing the level of prepulse reduced the stability of the electron beam as observed in our earlier investigations [14].

It is well recognized from the number of experimental, theoretical, and simulation studies that a well-collimated quasimonoenergetic electron beam could be produced from a self-injected laser wakefield acceleration under a wide range of laser and plasma parameters that were eventually attributed to the acceleration in the blowout (or bubble) regime [1]. The high-quality electron beam observed in the present experiment indicates laser wakefield acceleration in the blowout regime could be possible through strong self-modulation and/or self-evolution of the laser pulse in the high density plasma. In the blowout regime, transverse self-injection of electrons into the 3D wakefield occurs for 
$a_{0} \gtrsim 3$, and these electrons get accelerated and achieve energy gain $W_{\text {gain }}=(2 / 3)\left(n_{c} / n_{e}\right) a_{o} m_{o} c^{2}$ limited by the dephasing length [2]. For the plasma density $n_{e} \simeq$ $3.6 \times 10^{19} \mathrm{~cm}^{-3}$, where self-injected electrons were detected, relativistic self-focusing was also observed, as expected, since $P_{L} / P_{c} \gtrsim 3.7$, where $P_{c}(\mathrm{GW}) \simeq 17\left(n_{c} / n_{e}\right)$ is the critical power for self-focusing [15] (where GW stands for gigawatts). For the above density range, the plasma wavelength $\lambda_{p}(\mu \mathrm{m}) \simeq 10.5 \times n_{e}^{-0.5}\left(\times 10^{19} \mathrm{~cm}^{-3}\right) \lesssim$ $5.5 \mu \mathrm{m}$ is smaller in comparison to the laser pulse length $\left(c \tau_{L}=13.5 \mu \mathrm{m}\right)$ and the focal spot $\left(w_{0}=10 \mu \mathrm{m}\right)$ by a factor $\gtrsim 2.5$ and $\gtrsim 1.8$, respectively. Although the initial laser pulse parameters do not satisfy the conditions for matched spot $\left(w_{0} \simeq \lambda_{p}\right.$ and $\left.c \tau_{L} \simeq \lambda_{p} / 2\right)$ and self-injection $\left(a_{0} \gtrsim 3\right)$ immediately upon entry into the plasma, all of the above parameters might evolve nonlinearly over some initial interaction length in the plasma through self-focusing and self-compression of the laser pulse due to refractive index gradients of the plasma wave, eventually leading to the self-injection of electrons as observed in a number of earlier experiments and simulations under a wide range of laser and plasma parameters $[1,16,17]$. If one assumes that the compressed beam waist tends towards $\lambda_{p}$, and the compressed pulse length towards $\lambda_{p} / 2$, an expression $a_{0, \text { eff }}=6.55 \times 10^{-6} \cdot \sqrt{\frac{n_{e}}{n_{c}} \cdot \frac{E_{L}(J)}{\tau_{L}(s)}}$ for effective $a_{o}$ can be obtained from the relation $\frac{P_{L}}{P_{C}} \simeq \frac{\pi^{2}}{8} a_{0}^{2} \frac{w_{0}^{2}}{\lambda_{p}^{2}}$. For $n_{e} \sim 3.6 \times 10^{19} \mathrm{~cm}^{-3}, \tau_{L}=\lambda_{p} / 2 c$, and $E_{L}=145 \mathrm{~mJ}$, one gets $a_{0, \text { eff }} \simeq 3.8$, which agrees well with the findings of the self-injection threshold in earlier experiments, theory, and 3D particle-in-cell (PIC) simulations [2,16,17]. The observed low charge electrons just near the threshold density could be due to relatively slow evolution at lower density, which results in the matched conditions being reached close to the end of the interaction length. This would result in the onset of self-injection close to the end of the interaction length, leading to the production of lowenergy and low-charge electrons due to the small acceleration length available. However, at a higher density of $5.8 \times 10^{19} \mathrm{~cm}^{-3}$, the pulse evolution could be sufficiently fast so that the self-injection occurs well before the end of the interaction length, providing sufficient length for the generation of a high-energy quasimonoenergetic electron beam. The energy expected according to the expression for $W_{\text {gain }}$ at $5.8 \times 10^{19} \mathrm{~cm}^{-3}$ is $54 \mathrm{MeV}$, considering matched $a_{0, \text { eff }}=5.4$ estimated for this density. The measured energy $35.6_{-2.5}^{+3.9} \mathrm{MeV}$ is reasonably close to the expected value, considering that the expression for $a_{0, \text { eff }}$ is based on simple assumptions. The predicted value might be on the higher side due to a slightly higher value of $a_{0 \text {,eff }}$ estimated based on the assumption that all of the energy is trapped and compressed, which is less likely at high plasma density due to loss of a fraction of energy in self-modulation during the initial phase of interaction and also due to reduced coupling of the laser energy to the bubble due to a nonideal focal spot [10]. The difference may also arise when the length of acceleration from the point of self-injection is shorter than the dephasing length. Interestingly, according to the energy scaling in the linear regime, $W_{\text {gain }}=2 \gamma_{\phi}^{2} m_{o} c^{2}\left(E_{z} / E_{0}\right)$, where $\gamma_{\phi}^{2}=n_{c} / n_{e}$, the expected energy of electron beam would be $\sim 30 \mathrm{MeV}\left(\right.$ at $\left.n_{e}=5.8 \times 10^{19} \mathrm{~cm}^{-3}\right)$ assuming $E_{z}=E_{0}$.

Generation of quasimonoenergetic electron beams with virtually no low-energy background may be attributed to the self-injection just above the threshold condition, which ensures termination of the self-injection process very quickly. The near-threshold condition was verified from the observation that a decrease in plasma density by about $10 \%$ from $5.8 \times 10^{19} \mathrm{~cm}^{-3}$ resulted in a reduction of the electron beam charge and reproducibility significantly. Our observation of the deterioration of electron beam divergence at higher plasma density agrees well with the results of a recent report on the generation of background-free stable electron beams due to near threshold injection in the blowout regime by Banerjee et al. [10] using much higher power $(\sim 40 \mathrm{TW})$ laser pulses and lower plasma density $\leq 1 \times 10^{19} \mathrm{~cm}^{-3}$. Although we have not measured the electron energy spectrum at higher plasma density in the present study, it may be expected that increasing density would result in deterioration of the electron energy spectrum as observed in Ref. [10]. Although the near-threshold operation of the accelerator limits the beam charge to a relatively lower value, it significantly improves the electron beam quality due to a negligible beam loading effect [18]. The measured charge of few $\mathrm{pC}$ is much smaller compared to the charge $\sim 100 \mathrm{pC}$ predicted by the scaling law of the blowout regime, based on the beam loading effect [2]. This provides additional confirmation to the idea that the self-injection is limited by the near-threshold injection process but not by the beam loading effect. The observed ellipticity of the electron beam may be attributed to the interaction of injected electrons with the transverse laser electric field, resulting in momentum gain along the horizontal or polarization direction [19]. The stronger transverse focusing force of the bubble at higher plasma densities could make the defocusing force due to the laser field less significant at higher plasma density, which might explain the lower ellipticity of the electron beam observed in the present experiment. It may be noted that although the simple model based on assumptions on laser pulse evolution [17] aids to grossly understand the physics aspects of high-quality electron beam generation, a more accurate and detailed understanding of laser pulse evolution and wakefield acceleration dynamics in this new regime requires investigation using PIC simulations. In a very recent PIC simulation and experimental study using 60 fs duration laser pulse with power $<2$ TW and intensity $\simeq 2 \times$ $10^{17} \mathrm{~W} / \mathrm{cm}^{2}$ [20], a high-quality electron beam with sub-MeV energy was observed from self-modulated laser 
wakefield acceleration by a weak-contrast intense femtosecond laser pulse interaction with a prepulse formed plasma on a metal foil surface in a regime similar to our experimental condition $\left(c \tau_{L} \gg \lambda_{p}\right)$ and density. Their PIC simulations showed pulse steepening and selfmodulation of the laser pulse leading to intense plasma wave and subsequent generation of a quasimonoenergetic electron beam.

Self-injection based stable quasimonoenergetic electron beam generation was earlier reported at $n_{e}=5 \times$ $10^{19} \mathrm{~cm}^{-3}$ in the regime $c \tau_{L} \simeq 2 \lambda_{p}$ using 10 times higher power compared to that in the present experiment [21]. However, the beam was reported to have a background halo, in contrast to the observation in the present experiment. A background-free, quasimonoenergetic electron beam with peak energy $\lesssim 25 \mathrm{MeV}$ was reported in a previous experiment at $n_{e}=2 \times 10^{19} \mathrm{~cm}^{-3}$ in the regime $c \tau_{L}<\lambda_{p} / 2$ using 8 fs laser pulses of 5 TW power [9]. However, stability of the beam was not discussed. One important aspect of this experiment was that the interaction length was only about $100 \mu \mathrm{m}$, as the pulse did not require much evolution before triggering self-injection. In the present experiment, however, a few hundred $\mu \mathrm{m}$ interaction length, combined with the high density, might have facilitated sufficient pulse evolution, eventually leading to the self-injection and acceleration in the bubble regime. We have observed quasimonoenergetic beams with lower energy $\sim 20 \mathrm{MeV}$ at relatively higher plasma density of $8.5 \times 10^{19} \mathrm{~cm}^{-3}$ in our previous experiment using the similar laser intensity but with almost double the focal spot size [12]. In that experiment, the wakefield excitation and the electron acceleration occurred as a result of strong selfmodulation seeded by the forward Raman scattering instability [22], which is inherently susceptible to shot-to-shot fluctuations. It was noted in another previous experiment that an amplified spontaneous emission prepulse with sufficient intensity and duration forms preplasma that helps to guide the main laser beam in the low density plasma channel and accelerate the electrons to higher energies [14]. However, the inherent shot-to-shot variation of the prepulse parameters causes fluctuation in electron beam parameters. Although the electron energy in the present study is relatively less compared to Ref. [14], a low level of prepulse in the present study is found to contribute to improvement in the stability of the electron beam parameters as observed in an earlier study [23].

In summary, a stable, background-free, quasimonoenergetic electron beam has been demonstrated from selfinjected laser wakefield acceleration at high plasma density using a sub-10 TW power, 45 fs duration Ti:sapphire laser. The experimental results open a way to easily use energetic electron beams produced with small laser facilities for applications. The high-quality electron beam generation indicates near threshold self-injection and acceleration of electrons in the laser wakefield excited in the blowout regime, despite the fact that the initial laser pulse length is 3 times the plasma wavelength. This has been explained considering the significant evolution of the laser pulse due to self-modulation and self-evolution during the initial phase of the interaction. However, an accurate and detailed understanding of physics of laser pulse evolution and wakefield acceleration dynamics requires PIC simulations, which will be the subject of our future study.

The authors acknowledge the technical support by R. A. Khan, R. A. Joshi, R. K. Bhat, and S. K. Nagar, and mechanical support by R. P. Kushwaha, S. Sebastin, and K. C. Parmar during the experiment.

[1] E. Esarey, C. B. Schroeder, and W. P. Leemans, Rev. Mod. Phys. 81, 1229 (2009) and references therein.

[2] W. Lu, M. Tzoufras, C. Joshi, F. S. Tsung, W. B. Mori, J. Vieira, R. A. Fonseca, and L. O. Silva, Phys. Rev. ST Accel. Beams 10, 061301 (2007).

[3] V. Malka, J. Faure, Y. Gauduel, E. Lefebvre, A. Rousse, and K. T. Phuoc, Nat. Phys. 4, 447 (2008).

[4] J. Faure, C. Rechatin, A. Norlin, A. Lifschitz, Y. Glinec, and V. Malka, Nature (London) 444, 737 (2006).

[5] B. B. Pollock, C. E. Clayton, J.E. Ralph, F. Albert, A. Davidson, L. Divol, C. Filip, S. H. Glenzer, K. Herpoldt, W. Lu, K. A. Marsh, J. Meinecke, W. B. Mori, A. Pak, T. C. Rensink, J. S. Ross, J. Shaw, G. R. Tynan, C. Joshi, and D. H. Froula, Phys. Rev. Lett. 107, 045001 (2011).

[6] A. J. Gonsalves, K. Nakamura, C. Lin, D. Panasenko, S. Shiraishi, T. Sokollik, C. Benedetti, C. B. Schroeder, C. G. R. Geedes, J. Van Tilborg, J. Osterhoff, E. Esarey, C. Toth, and W. P. Leemans, Nat. Phys. 7, 862 (2011).

[7] A. Buck, J. Wenz, J. Xu, K. Khrennikov, K. Schmid, M. Heigoldt, J. M. Mikhailova, M. Geissler, B. Shen, F. Krausz, S. Karsch, and L. Veisz, Phys. Rev. Lett. 110, 185006 (2013).

[8] S. Y. Kalmykov, A. Beck, X. Davoine, E. Lefebvre, and B. A. Shadwick, New J. Phys. 14, 033025 (2012).

[9] K. Schmid, L. Veisz, F. Tavella, S. Benavides, R. Tautz, D. Herrmann, A. Buck, B. Hidding, A. Marcinkevicius, U. Schramm, M. Geissler, J. Meyer-ter-Vehn, D. Habs, and F. Krausz, Phys. Rev. Lett. 102, 124801 (2009).

[10] S. Banerjee, S. Y. Kalmykov, N. D. Powers, G. Golovin, V. Ramanathan, N. J. Cunningham, K. J. Brown, S. Chen, I. Ghebregziabher, B. A. Shadwick, and D. P. Umstadter, Phys. Rev. ST Accel. Beams 16, 031302 (2013).

[11] T. Hosokai, K. Kinoshita, T. Watanabe, K. Yoshii, T. Ueda, A. Zhidkovand, M. Uesaka, Proceedings of the 8th European Particle Accelerator Conference, Paris, 2002 (EPS-IGA and CERN, Geneva, 2002) p. 981.

[12] B. S. Rao, A. Moorti, P. A. Naik, and P. D. Gupta, New J. Phys. 12, 045011 (2010).

[13] Y. C. Wu, B. Zhu, K. G. Dong, Y. H. Yan, and Y. Q. Gu, Rev. Sci. Instrum. 83, 026101 (2012).

[14] B. S. Rao, J. A. Chakera, P. A. Naik, M. Kumar, and P. D. Gupta, Phys. Plasmas 18, 093104 (2011).

[15] G. Z. Sun, E. Ott, Y. C. Lee, and P. Guzdar, Phys. Fluids 30, 526 (1987). 
[16] F. S. Tsung, W. Lu, M. Tzoufras, W. B. Mori, C. Joshi, J. M. Vieira, L. O. Silva, and R. A. Fonseca, Phys. Plasmas 13, 056708 (2006).

[17] S. P. D. Mangles, A. G. R. Thomas, O. Lundh, F. Lindau, M. C. Kaluza, A. Persson, C.-G. Wahlstrom, K. Krushelnick, and Z. Najmudin, Phys. Plasmas 14, 056702 (2007).

[18] C. Rechatin, X. Davoine, A. Lifschitz, A. B. Ismail, J. Lim, E. Lefebvre, J. Faure, and V. Malka, Phys. Rev. Lett. 103, 194804 (2009).

[19] S. P. D. Mangles, A. G. R. Thomas, M. C. Kaluza, O. Lundh, F. Lindau, A. Persson, F. S. Tsung, Z. Najmudin, W. B. Mori, C.-G. Wahlstrom, and K. Krushelnick, Phys. Rev. Lett. 96, 215001 (2006).

[20] Yu. A. Malkov, A. N. Stepanov, D. A. Yashunin, L. P. Pugachev, P. R. Levashov, N. E. Andreev, K. Yu. Platonov, and A. A. Andreev, High Power Laser Sci. Eng. 1, 80 (2013).

[21] S. M. Wiggins, R. C. Issac, G. H. Welsh, E. Brunetti, R. P. Shanks, M. P. Anania, S. Cipiccia, G. G. Manahan, C. Aniculaesei, B. Ersfeld, M. R. Islam, R. T. L. Burgess, G. Vieux, W. A. Gillespie, A. M. MacLeod, S. B. van der Geer, M. J. de Loos, and D. A. Jaroszynski, Plasma Phys. Controlled Fusion 52, 124032 (2010).

[22] B. Hidding, M. Geissler, G. Pretzler, K.-U. Amthor, H. Schwoerer, S. Karsch, L. Veisz, K. Schmid, and R. Sauerbrey, Phys. Plasmas 16, 043105 (2009).

[23] S. P. D. Mangles, A. G. R. Thomas, M. C. Kaluza, O. Lundh, F. Lindau, A. Persson, Z. Najmudin, C.-G. Wahlstrom, C. D. Murphy, C. Kamperidis, K. L. Lancaster, E. Divall, and K. Krushelnick, Plasma Phys. Controlled Fusion 48, B83 (2006). 